\title{
IL PENSIERO DEI REGOLATORI
}

\author{
GIUSEPPE SOPRANZETTI (*)
}

SunTO. - Nella recente, lunga crisi le banche sono state al centro di un crescente attacco. La crisi è stata, peraltro, di una tale portata che è ragionevole pensare che non possano esserci "attori economici" esenti da responsabilità. Alle banche è comunque ora richiesto un radicale mutamento delle strategie per reagire agli straordinari cambiamenti che, da più di un ventennio, stanno radicalmente mutando il contesto di riferimento. La crisi, unita ai profondi mutamenti normativi, ha fatto saltare l'equilibrio che in Italia aveva caratterizzato il rapporto - probabilmente immaturo - banca/impresa e che aveva prodotto un sistema con caratteristiche prevalentemente "bancocentriche". È necessario trovare nuovi equilibri attraverso un rapporto banca/impresa più maturo e trasparente. Sarà sempre più necessaria una visione olistica del sostegno alla crescita, unendo le forze in campo, con banche ed imprese che assumono il ruolo di due vogatori nello stesso scafo e con la concreta possibilità che salga anche il "mercato" (azioni, obbligazioni), come terzo membro dell'equipaggio. L'Unione Bancaria - importante, ulteriore passo verso un Europa federale - potrebbe però rappresentare un rischio nel caso in cui prevalgano i pregiudizi e non si realizzi un "campo di gioco" (fiscale, contabile, ecc.) completamente allineato. Serve un'Europa matura e solidale, aliena da pregiudizi, che ritrovi lo spirito dei padri fondatori.

$$
* * *
$$

ABSTRACT. - During the recent, long crisis, banks have been at the center of growing attacks. The crisis has been of such a magnitude, though, that it is reasonable to think that no "economic entity" may be exempt from liability. Today, nevertheless, banks are required a radical revision of strategies, in order to respond to the extraordinary changes that, since more than twenty years, are radically modifying the frame of reference. In Italy, the crisis and the profound changes in the banking laws have blasted the balance characterizing the relationship between banks and enterprises; a balance probably immature, that has generated a system predominantly "centered on the banks". Now it is necessary to find a new equilibrium, based on more mature and transparent relations. Moreover, it is increasingly necessary to foster a holistic perspective aimed at supporting growth; banks and enterprises should join forces, taking on the role of two rowers in the same boat, and the "market"

(*) Banca d'Italia, Sede di Milano, Italia.

E-mail: giuseppe.sopranzetti@bancaditalia.it 
(stocks, bonds) should get on board too, as third member of the crew. In this context the Banking Union, though representing an important further step towards a federal Europe, could even pose a risk, if prejudices prevail and a complete "level playing field" (tax, accounting, etc.) is not realized. Therefore, we need a mature and supportive Europe, devoid of preconceptions and able to rediscover the spirit of the founding fathers.

Un sentito ringraziamento all'Istituto Lombardo Accademia di Scienze e Lettere, ed in particolare al suo Presidente Silvio Beretta e a Roberto Ruozi, che mi hanno voluto coinvolgere in questa iniziativa che si caratterizza per la presenza di un parterre di relatori tale da mettere quasi in soggezione.

Debbo però aggiungere che tale stato d'animo è immediatamente superato dalla ironica simpatia del titolo del nostro incontro, che mi fa intuire che sul tema possiamo confrontarci senza tante formalità e con la massima trasparenza.

Vorrei, pertanto, utilizzare un approccio che tenti di cavalcare l'ironia del titolo del nostro incontro, portando considerazioni ispirate dalla realtà in cui lavoro da 35 anni, ma la cui responsabilità è strettamente personale.

Inizio, pertanto, citando, un intervento che feci in apertura del convegno annuale ACRI ASSBANK, tenutosi a Milano due anni fa. In quella circostanza ricordai all'autorevole parterre che indubbiamente le banche non stavano attraversando un bel momento quanto ad immagine.

Nella complessa crisi che stavamo vivendo - e che speriamo di esserci lasciati alle spalle - le banche erano state al centro di un costante, crescente attacco alla ricerca - a mio giudizio e con un tipico meccanismo di semplificazione e fuga dall'analisi - di un facile capro espiatorio, cui attribuire le principali responsabilità della crisi.

Crisi, invece, di portata tale che difficilmente si può pensare che ci siano attori economici esenti da responsabilità (le responsabilità sono indubbiamente diffuse anche se con ponderazioni, ovviamente, diverse).

Per supportare tale affermazione - oltre a sottolineare che avevo da poco partecipato ad un convegno a Varese dall'emblematico titolo: Conosci il tuo nemico, e indovinate a chi si riferiva il titolo? - utilizzai un brano tratto da un libro nel 2012 del noto psichiatra Vittorino Andreoli (Il denaro in testa), che nelle prime pagine, cercando di interpretare un pensiero diffuso, afferma:

Le banche... hanno rovinato l'economia e portato al tracollo non singoli paesi, ma tutto l'Occidente. 
Naturalmente hanno perduto la faccia e di sicuro la dimensione (quasi) sacra di cui godevano (sino a quel momento infatti le "Banche erano percepite come luoghi di sacralità e si mostravano con la magnificenza tipica delle cattedrali, luoghi del Signore").

Consapevoli di aver perduto la sacralità...

prosegue Andreoli

le banche vogliono ora recuperare e si impegnano, soprattutto, in un lifting, che per loro è prevalentemente rappresentato dalla comunicazione.

Il rischio, che Andreoli individua per le banche, è però quello di apparire come una prostituta che passa dal chirurgo per un "punto d'oro" e poi cerca di farsi sposare da un babbeo, illudendolo di essere stato con una verginella.

Citazione indubbiamente forte ma, a mio giudizio, nei momenti di difficoltà è meglio affrontare di petto i problemi e non girarci attorno.

Pochi giorni dopo mi capitò di confrontarmi sulle affermazioni di Andreoli con alcuni colleghi, che prontamente osservarono: "l'autore si riferisce ovviamente agli intermediari prevalentemente attivi nei mercati anglosassoni attori/vittime della prima crisi finanziaria"?

Purtroppo non è così! Il libro - come spesso l'opinione pubblica tende a fare di tutta l'erba un fascio, senza distinguere tra banche anglosassoni, tedesche, spagnole, olandesi etc... e banche italiane, che (in quanto più tradizionali, più attentamente vigilate e con clienti con una elevata propensione al risparmio) erano comunque uscite a testa alta dalla iniziale crisi finanziaria... anche se poi avevano incrociato i pericolosi scogli dei debiti sovrani e sono poi state inevitabilmente coinvolte nei processi recessivi.

Il libro Intermezzo dell'amico professor Ruozi fornisce un efficace panorama delle banche internazionali coinvolte nella crisi finanziaria.

Restando aderenti al tema dell'odierno incontro, l'episodio che ho raccontato è da ascrivere sicuramente al profilo "Banche croce dell'economia e della società".

$\mathrm{Ma}$, sempre su tale profilo e passando dalla letteratura all'esperienza vissuta, consentitemi di ricordare due esempi risalenti alla mia esperienza siciliana, isola dove ho lavorato dal 2005 al 2011, alla direzione prima della Filiale di Catania e poi della Sede di Palermo.

Ricorderete sicuramente che già nel 2009 fu lanciato - da parte dell'allora Ministro dell'Economia - l'allarme sul credit crunch, e furono costituiti, a tale proposito, presso le Prefetture gli Osservatori sulle dinamiche del credito, per vigilare "sulle banche...che non erogavano credito". 
La relazione finale dell'Osservatorio presso la Prefettura di Palermo concluse i suoi lavori rilevando che, a giudizio delle imprese, erano molto più gravi i ritardi nei pagamenti da parte delle PP.AA. rispetto alla cautela che si stava progressivamente evidenziando nell'erogazione del credito da parte delle banche.

Su un altro fronte, il predecessore di Crocetta alla Presidenza della Regione Siciliana - Raffaele Lombardo (condannato nel 2014 in primo grado per concorso esterno in associazione mafiosa) - era solito affermare, sul finire del decennio scorso, che il mancato decollo economico dell'isola era da imputare al fatto che le banche presenti in regione non fossero più siciliane ma acquisite, dopo le diffuse crisi bancarie degli anni '90, da banche del Nord.

Era anche qui facile affermare - alla luce di numerosi studi su credito e crescita economica nel Mezzogiorno d'Italia - che di fronte ai gravi problemi della Sicilia il credito era un piccolo problema.

Le principali questioni alla base del mancato sviluppo economico dell'isola sono sicuramente altre!

Da quanto sin qui detto emerge un quadro dove la "croce" sembra indubbiamente prevalere sulla "delizia".

In questo clima mi sono più volte trovato costretto, nel corso di convegni, a cercare di "riportare la palla al centro", stigmatizzando preliminarmente che la Banca d'Italia non è certo il difensore d'ufficio delle banche... anzi, siamo però cultori dell'analisi e rifuggiamo, per metodo, dall'affannosa ricerca di capri espiatori, che prescindono dall'approfondimento di dati e informazioni.

Le banche non meritano pertanto di essere crocefisse, anche se, come per tutti gli altri attori economici, molto c'è da lavorare in conseguenza degli straordinari cambiamenti che hanno caratterizzato gli ultimi due decenni, sfociati nella "guerra dei 7 anni". Ma su queste considerazioni, se ci sarà tempo, tornerò fra poco.

C'è indubbiamente, tuttavia, l'esigenza da parte delle banche di un recupero della fiducia della clientela e, soprattutto, dell'opinione pubblica.

Non ha, infatti, certamente aiutato un approccio delle banche, sul fronte della trasparenza, non del tutto aperto nei riguardi della clientela, come si è potuto constatare nei rafforzati controlli, allorché dal 2010 la tutela del cliente è stata affidata normativamente alla Banca d'Italia.

Ma veniamo alla "delizia", citata nel titolo del convegno.

Qui incontriamo subito un evidente paradosso: ovvero la rilevata avversione nei confronti delle banche avviene in un Paese ad economia prevalentemente bancocentrica. 
In un paese cioè dove il credito bancario rappresenta oltre il $90 \%$ del PIL e il $64 \%$ dei debiti complessivi delle imprese, contro una media dell'area euro del $46 \%$.

Delizia, pertanto, perché senza un sistema bancario così impegnato a sostenere le imprese, allo stato dell'arte sarebbe difficile fare investimenti e conseguentemente perseguire sentieri di crescita dell'economia.

$\mathrm{Ma}$ è da iscrivere dal lato della delizia anche il fatto che in questi anni le banche italiane hanno attraversato la crisi senza che ci sia stata l'esigenza di accedere a massicce ricapitalizzazioni con fondi pubblici.

In altre parole ai contribuenti italiani non sono stati addossati gli elevati costi per perdite e fallimenti bancari osservati altrove: Germania 1,8\% del PIL, Belgio 4,3\%, Paesi Bassi 5,1\%, Spagna 5,5\%.

Ma allora viene spontaneo domandarsi: che cosa ha fatto rompere quell'equilibrio che esisteva nei rapporti banca/impresa e che aveva alimentato un così rilevante peso dei finanziamenti bancari nei bilanci delle imprese?

Tre sono - a mio giudizio - gli straordinari mutamenti del contesto in cui operano le banche che hanno alterato gli equilibri esistenti: due mi piace chiamarli "catastrofi innaturali", il terzo è rappresentato dai mutamenti normativi (anch'essi di straordinaria intensità).

Il mercato creditizio è stato coinvolto negli ultimi anni da due catastrofi innaturali per entità e durata: la prima l'abbiamo già ricordata (la consistente perdita di reputazione), la seconda è rappresentata dalle straordinarie difficoltà derivanti dalle crisi (finanziaria, debito sovrano e recessione), le cui implicazioni sono sotto gli occhi di tutti (in termini di rischio di credito, redditività...).

Nel contempo le banche sono state interessate da una continua e profonda evoluzione del contesto normativo - tuttora in corso - conseguenza e reazione, in buona parte, delle vicende alla base di tali catastrofi e volta ad aumentare i margini di sicurezza per fronteggiare eventuali, ulteriori crisi attraverso maggiori dotazioni patrimoniali e di liquidità.

Gli interventi normativi hanno avuto altresì l'obiettivo di trasferire il costo delle crisi bancarie dal settore pubblico ai finanziatori delle banche (azionisti e detentori di altre passività).

Rilevano in proposito le norme di Basilea 3, la Direttiva BRRD (Bank Recovery and Resolution Directive), le proposte del FSB relative al TLAC (Total Loss Assorbing Capacity) e, in alcuni paesi, i vincoli allo svolgimento di talune attività.

La crisi e l'evoluzione normativa hanno quindi, come ben sappiamo, pesantemente inciso proprio sul fronte dei finanziamenti bancari. Hanno 
mostrato i limiti di un sistema che, pur con molte contraddizioni, aveva indubbiamente funzionato.

Prima della crisi il nostro Paese era infatti caratterizzato da un contesto - di condizioni di mercato e normative - in cui era facile erogare credito facendo, ad esempio, funding sui mercati esteri all'ingrosso, in presenza di una consistente liquidità che circolava velocemente sorretta dal fattore fiducia.

Inoltre, la bassa rischiosità dei prenditori - e il contesto normativo non richiedevano ingenti capitali proprio a fronte dei prestiti.

Cambiati i paradigmi preesistenti, per le banche è stato razionale, nell'interesse anche dei risparmiatori, rallentare o ridurre in questi anni il credito alle imprese e aumentare altre forme di investimento. D'altra parte anche la domanda di credito è necessariamente diminuita nel corso della crisi.

È stato analizzato (da Salvatore Rossi Direttore Generale della Banca d'Italia) se in tale atteggiamento restrittivo vi fosse colpa da parte delle banche, se vi fosse cioè l'incapacità delle stesse di valutare con sapienza e lungimiranza il merito creditizio dei singoli imprenditori (problema di efficienza allocativa).

Il giudizio non può che essere articolato, ma vi sono stati certamente comportamenti eterogenei, fermo restando comunque che nel nostro Paese ha operato e opera un intreccio perverso tra caratteristiche strutturali del sistema delle imprese (piccole, familiari, spesso opache, fragili finanziariamente) e del sistema finanziario.

Tale situazione determina un "fallimento del mercato": i soggetti privati, debitori e creditori, non riescono cioè a far funzionare efficientemente il mercato del credito perché sono mossi ciascuno da incentivi e disincentivi in conflitto con l'interesse generale.

L'impresa "piccola" (le PMI rappresentano l'asse portante del nostro tessuto economico) intende il più possibile restare opaca; è addirittura abituata a sottorappresentare la propria situazione reddituale per timore del fisco e difficilmente si apre al mercato proprio perché troppo piccola o, soprattutto, per il timore di perdere il controllo familiare.

La banca, a volte, non si dota si strutture valutative adeguate perché trova più comodo, e meno costoso nell'immediato, fidarsi delle relazioni personali.

Il risultato è la selezione avversa e il razionamento del credito. Occorre spezzare questo circolo vizioso dove, alla fine, tutti perdono!

$\grave{E}$, pertanto, necessario che le banche affinino gli strumenti qualiquantitativi per la selezione del credito. In particolare, le banche grandi 
dovranno sempre più approfondire la qualità dei progetti; le banche piccole dovranno affiancare alla conoscenza diretta dei richiedenti credito più compiute analisi quantitative.

Le imprese manifatturiere dovranno aprirsi di più alle banche, instaurando un rapporto più stretto e maturo, e al mercato (azioni e obbligazioni).

Intermediari e imprese non bancarie dovranno sempre più acquisire quella che chiamerei "visione olistica" del sostegno alla crescita, con un'unione delle forze, un salto culturale, passando da un sistema di banche/imprese in costante contrapposizione (due vogatori sulla stessa imbarcazione) ad uno caratterizzato dalla concreta possibilità che salga il terzo vogatore, cioè il mercato.

È questo un passaggio obbligato: è, infatti, indubbio che l'indebitamento e la dipendenza dal credito bancario sono segnali di vulnerabilità per le imprese italiane.

Il credito bancario adempie ai propri compiti, con il necessario concorso di capitali direttamente investiti dalle imprese e con le risorse che possono essere utilmente raccolte dal mercato.

Una dotazione di capitale più elevata agevolerebbe l'accesso delle imprese al credito; insieme ad una maggiore diversificazione delle fonti di finanziamento, le renderebbe più robuste.

Il Libro Verde della Commissione Europea sulla Capital Markets Union, invita le banche - primo collegamento delle PMI con il mercato dei capitali - a dare supporto alle PMI nello sviluppo di nuove forme di finanziamento.

Riforme strutturali volte a rilanciare la crescita agevolerebbero il reperimento di capitale di rischio e incoraggerebbero gli imprenditori a impegnare risorse proprie, mostrando fiducia nelle prospettive delle loro aziende.

Sin qui ho cercato di analizzare alcuni dei motivi per i quali le banche hanno questo ruolo di croce e delizia dell'economia e della società, lasciando per lo più sullo sfondo le implicazioni provenienti dall'avvio dell'Unione Bancaria.

In chiusura, vorrei sottolineare come gli sviluppi dell'Unione Bancaria potrebbero tuttavia far spostare il nostro pendolo (croce-delizia) pericolosamente dalla parte della croce.

L'Unione Bancaria è un'importante, ulteriore tappa per realizzare quell'Europa unita senza la quale le nazioni di questo continente rischierebbero la progressiva emarginazione nell'ambito dei nuovi contesti risultanti dalla globalizzazione.

È un passaggio essenziale, tra l'altro, per rompere il legame tra debito 
sovrano e debito bancario, che grande responsabilità ha avuto nell'alimentare una parte della recente crisi.

È un passaggio obbligato per livellare il campo di gioco delle banche operanti in Europa, pur rimanendo, comunque, condizione necessaria ma non sufficiente.

Infatti, molte sono le differenze e molti sono i pregiudizi. È pertanto necessario superare le differenze (fiscali, criteri contabili, etc.) ed eliminare i pregiudizi, neutralizzandoli in quello che dovrebbe essere l'autentico spirito europeo.

Serve un'Europa più matura e solidale, aliena da pregiudizi, che ritrovi lo spirito dei padri fondatori. Occorre evitare l'errore di dimenticarsi delle ragioni profonde dell'Unione e degli errori del passato, allorché una veduta corta ha creato i presupposti per ulteriori, pericolose divisioni.

È altresì importante, ora che emergono segnali di ripresa, fare tesoro degli insegnamenti della crisi, evitando politiche pro-cicliche.

Nella quantificazione dei presìdi di capitale, ad esempio, vanno necessariamente contemperate diverse esigenze:

- garantire regole certe relativamente ai criteri sottostanti, in modo da consentire alle banche un'adeguata pianificazione;

- assicurare che le dotazioni patrimoniali proteggano il singolo intermediario dalle proprie vulnerabilità e rassicurino i mercati sulla solidità delle banche dell'area;

- valutare con attenzione gli effetti macroeconomici che ulteriori richieste di capitale potrebbero avere sull'offerta complessiva di credito all'economia, tenendo anche conto dei progressi già conseguiti dalle banche sul fronte patrimoniale e dell'attuale fase ciclica.

Tutte queste esigenze debbono trovare composizione nella discussione all'interno del Consiglio di Vigilanza e nelle decisioni del Consiglio Direttivo della BCE.

Il passaggio al nuovo equilibrio caratterizzato da banche dotate di più capitale e più liquidità va attuato con gradualità e lungimiranza, valutando i rischi di un aggiustamento repentino.

L'attività di supervisione micro-prudenziale deve confrontarsi con le esigenze di carattere macro-prudenziale. Occorre mirare a conferire una forza non effimera al sistema finanziario, coniugando le esigenze di stabilità delle banche con quelle della ripresa produttiva.

È assolutamente necessario superare le differenze (fiscali, norme societarie e fallimentari) ed eliminare i pregiudizi, ripristinando l'autentico spirito europeo dei padri fondatori. 
Avviandomi alla conclusione vorrei parlare di guerra... ricordando che il lungo periodo di crisi che abbiamo attraversato è stato definito da Salvatore Rossi "la guerra dei sette anni".

Mi è utile, a tale scopo, parafrasare Keynes, che nel 1919 scrisse Le conseguenze economiche della pace, ove evidenziò la sua contrarietà al Trattato con il quale si era conclusa la Conferenza di Pace di Parigi, a suo giudizio, foriero di pesanti conseguenze sul futuro politico, economico e sociale dell'Europa.

Secondo Keynes - terminata la guerra - un errore fu quello di chiedere (ai perdenti) l'impossibile, sacrificando la sostanza all'apparenza e, alla fine, rischiando di perdere tutto.

Altro errore fu quello di concentrarsi troppo sul passato, invece di guardare al futuro. Sarebbe stato più importante e proficuo, a suo giudizio, vedere le cose in termini di umanità e civiltà europea in cerca di un nuovo ordine.

Detto in modo molto meno elegante, Keynes voleva forse dire che "a troppo tirare la corda...c'è poi il rischio che si spezzi”. Ė questo il sottile equilibrio che tutti gli attori in gioco debbono assolutamente avere ben presente.

Grazie dell'attenzione. 